\title{
The preparation of the poly(vinyl alcohol)/graphene nanocomposites with low percolation threshold and high electrical conductivity by using the large-area reduced graphene oxide sheets
}

\author{
T. N. Zhou, X. D. Qi, Q.Fu* \\ College of Polymer Science and Engineering, State Key Laboratory of Polymer Material Engineering, Sichuan University, \\ 610065 Chengdu, People's Republic of China
}

Received 24 March 2013; accepted in revised form 19 May 2013

\begin{abstract}
We report a method to prepare the poly(vinyl alcohol)/reduced graphene oxide (PVA/rGO) nanocomposites with low percolation threshold and high electrical conductivity by using the large-area reduced graphene oxide (LrGO) sheets. The large-area graphene oxide (LGO) sheets are expected to overlap better with each other and form the continuous GO network in PVA matrix than small-area graphene oxide (SGO). During the thermal reduction process, the LGO sheets are easily restored and improve the electrical conductivity of nanocomposites due to their low damage level of conjugate-structure. As a result, the percolation threshold of PVA/LrGO nanocomposites is $\sim 0.189 \mathrm{wt} \%$ lower than present reports $(0.5 \sim 0.7 \mathrm{wt} \%)$. At the LrGO content of $0.7 \mathrm{wt} \%$, the electrical conductivity of PVA/LrGO nanocomposites reaches $6.3 \cdot 10^{-3} \mathrm{~S} / \mathrm{m}$. Besides that, this method only takes $15 \sim 30 \mathrm{~min}$ to reduce the PVA/GO nanocomposites effectively.
\end{abstract}

Keywords: nanocomposites, poly(vinyl alcohol), large-area rGO, thermal reduction, electrical conductivity

\section{Introduction}

Conductive polymer composites have been used in a wide range of important technological applications, such as sensor, anti-static, microwave absorbing, and conductive coating. These composites are prepared by compounding the polymer and conductive nanoparticles (such as carbon black, carbon nanotubes, carbon fiber, graphite and metal particles) $[1,2]$. When the content of conductive nanoparticle reaches to a critical value (i.e. percolation threshold), a continuous electrical conductive network is formed by these conductive nanoparticles, rendring the polymer composite electrically conductive $[3,4]$ So the nanoparticles' electrical conductivity, dispersibility and the interaction with polymer matrix directly affect the property of the elec- trical conductive network and define the final electrical conductivity of polymer composites. According to the percolation theories, the percolation threshold depends on the filler shape, size and aspect ratio. A larger aspect ratio filler can more effectively decrease the percolation threshold of nanocomposites than a small one $[5,6]$. Besides that, the electrical conductivity of nanocomposites is also defined by the filler's pristine conductivity.

Graphene - a two-dimensional monolayer of $\mathrm{sp}^{2}$ carbon atom, is widely considered to be an ideal material for preparing the conductive polymer composites due to its high electrical conductivity $\left(10^{6} \mathrm{~S} / \mathrm{cm}\right)$, the high ratio of diameter to thickness and the excellent mechanical properties [7]. However, the strong $\pi-\pi$ interaction between graphene

\footnotetext{
${ }^{*}$ Corresponding author, e-mail: qiangfu@scu.edu.cn

(C) BME-PT
} 
sheets make them hardly dispersible in some organic solvents and polymer matrix homogenously, so it is difficult for them to form the continuous conductive network in a polymer matrix, especially at low graphene content. At present, the oxidation-reduction process is an efficient and common method to improve the dispersibility of graphene sheets in many polymer matrices and organic solvents. Firstly, making the graphene surface grafted with a lot of oxygen-functional groups by oxidation reaction, these polar groups increase the repellent force between graphene sheets and then separate them, so these separated graphene oxide (GO) sheets could be homogenously dispersed in many polymer matrices and organic solvents; the second step is to remove these polar groups in order to restore the electrical conductivity of GO sheets.

Due to the partial re-aggregation of the rGO sheets during the reduction processing, the surfactant agents or ultrasonic treatment are usually used to re-disperse the rGO sheets in the polymer matrix. After the reduction of GO sheets, the surfactant agents could form a coating layer on the surface of rGO sheets, those coating layer could make the separation of these sheets from each other and then helps the dispersion of the rGO sheets in the polymer matrix homogeneously. Due to the insulating properties of the surfactant agents, the coating layer would hinder the efficient charge transport, and decrease the final electrical conductivity of composites [8]. Besides that, these surfactant agents would play the role of the plasticization, and decrease the mechanical properties of composites. The ultrasonic treatment could assist the dispersion of nanofiller, but it would decrease the size of GO sheets and increase the structure defects of the GO sheets, these factors are disadvantageous for improving the final electrical conductivity of rGO sheets.

Our aim is to achieve the high electrical conductive polymer/rGO nanocomposite, so in our work we not only need using the large aspect ratio nanofiller, but also need optimizing the dispersion.

Zhao et al. [9] reported that the large area reduced graphene oxide (LrGO) sheets have lower intersheet contact resistance and less damaged conjugation structure level than that of small-area reduced graphene oxide ( $\mathrm{SrGO}$ ) sheets, and could be used to prepare the transparent conductive films with $840 \Omega / \mathrm{sq}$ at $78 \%$ transmittance, which was lower than that be made from the $\operatorname{SrGO}(19.1 \mathrm{k} \Omega / \mathrm{sq}$ at
$78 \%$ transmittance). So the LrGO sheets have following two advantages which are more suitable for improving the electrical conductivity of nanocomposites than the small one: 1) there are less intersheet junctions leading to the high intrinsic electrical conductivity, and 2) the large area is prone to form a continuous electrical conductive network. GO sheets could be reduced in different media such as water [10], ethanol, butanol, and ethylene glycol [11] at a special temperature, and the GO sheets also could be reduced in polymer matrix such as poly (vinylpyrrolidone), poly(vinyl acetate) [12] or polyvinylidene fluoride [13]. Glover et al. [12] reported that the GO sheets could be reduced in PVA matrix at the temperature range from 150 to $250^{\circ} \mathrm{C}$, and the result of Raman peak shift shown that the $\mathrm{C}: \mathrm{O}$ ratio is less than 10 after reduction. However, this method can maintain the good dispersion of rGO sheets and get rid of introducing the surfactant agents into polymer matrix $[14,15]$, meanwhile avoids using reducing agents, especially hazardous ones.

In this work, we use poly(vinyl alcohol) (PVA) as polymer matrix, and disperse the GO sheets in PVA matrix homogeneously through the interaction between the hydroxyl groups on the PVA chains and oxygen-functional groups located on the surface of GO sheets, and then to reduce the GO sheets through the in-situ thermal reduction at a special temperature which depends on the decomposition temperature of PVA $[12,13,16]$. In our method, there are two purposesintended, 1) usage of the LGO sheets to construct a continuous filler network, and 2) avoiding the introduction of reducing and surfactant agents during the reduction processing. The effect of electrical conductive network structure morphology on the final electrical conductivity of nanocomposites is studied through comparing the PVA/LrGO and PVA/SrGO nanocomposites.

\section{Experimental}

\subsection{Materials}

Artificial graphite power with an average size of $200 \mu \mathrm{m}$ were purchased from Qingdao Black Dragon graphite Co., Ltd, PVA was 99\% hydrolyzed with an average polymerization degree of 1700 (SINOPEC Sichuan vinylon works). Potassium permanganate $\left(\mathrm{KMnO}_{4}\right)$, sulfuric acid $\left(\mathrm{H}_{2} \mathrm{SO}_{4} 98 \%\right)$, hydrogen peroxide $\left(\mathrm{H}_{2} \mathrm{O}_{2}\right)$ and sodium nitrate $\left(\mathrm{NaNO}_{3}\right)$, sodium hydroxide $(\mathrm{NaOH})$, above-mentioned chemical agents were purchased from Kermel Chemical 
reagent plant (Tianjin, China). They were used as received.

\subsection{Preparation of the LGO and SGO sheets}

GO sheets were obtained by Hummer's method [17]. Graphite $(1 \mathrm{~g})$ was mixed with $\mathrm{NaNO}_{3}(1 \mathrm{~g})$ in $\mathrm{H}_{2} \mathrm{SO}_{4}(48 \mathrm{ml})$ at $0^{\circ} \mathrm{C}$ (ice bath), $\mathrm{KMnO}_{4}(6 \mathrm{~g})$ was slowly added in $1 \mathrm{~h}$. After that, the reaction mixture was first stirred at $0^{\circ} \mathrm{C}$ for $2 \mathrm{~h}$ and then at $30^{\circ} \mathrm{C}$ for $2.5 \mathrm{~h}$. Deionized water $(150 \mathrm{~mL})$ was slowly added to the reaction system and stirred for $1.5 \mathrm{~h}$ to obtain a graphite oxide suspension. The mixture was treated with $5 \% \mathrm{H}_{2} \mathrm{O}_{2}(50 \mathrm{~mL})$, filtered and washed with hot water until completely remove the residual salts and acids. For obtaining the LGO sheets, a low-speed centrifugation at $4000 \mathrm{rpm}$ was first used to remove thick multilayer flakes until all the visible particles were removed $(15 \mathrm{~min})$, then the supernatant was further centrifuged at $8000 \mathrm{rpm}$ for $7 \mathrm{~min}$ to separate precipitate (LGO) and supernatant, the SGO sheets were prepared by ultrasonication of abovementioned LGO sheets at the power of $100 \mathrm{~W}$ for $45 \mathrm{~min}$. The area distribution of LGO and SGO sheets was counted by scanning electron microscopy (SEM).

\subsection{Preparation of PVA/rGO nanocomposites}

The PVA/LGO and PVA/SGO nanocomposites with different filler contents were prepared as follows: PVA $(1 \mathrm{~g})$ was dissolved in deionized water $(10 \mathrm{~mL})$ at $98^{\circ} \mathrm{C}$ for $1 \mathrm{~h}$. The aqueous dispersion of the LGO or SGO sheets was gradually dropped into the PVA solution, and stirred at $98^{\circ} \mathrm{C}$ for $30 \mathrm{~min}$. No precipitate was observed in a few hours after magnetic stirring indicating that no aggregation of the LGO or SGO sheets occurred. The homogeneous PVA/ LGO or PVA/SGO solution was poured into an aluminum dish and kept at $60^{\circ} \mathrm{C}$ for $15 \mathrm{~h}$ to cast film. The thickness of the obtained films is about $0.1 \mathrm{~mm}$.

The PVA/LGO or PVA/SGO nanocomposites films were reduced through thermal reduction, firstly keep these samples in the furnace filled with $\mathrm{N}_{2}$, then increase the temperature of samples to reduction temperature at the rate of $10^{\circ} \mathrm{C} / \mathrm{min}$, and maintain the sample at special reduction temperature for $0,10,15$, and $30 \mathrm{~min}$ respectively. According to the weight ratio of GO (dry weight) to PVA, the reduction temperature and time, the nanocomposites films were denoted as PVA/LrGO- $x \mathrm{wt} \%-y^{\circ} \mathrm{C}-z$ min or
PVA/SrGO- $x$ wt $\%-y{ }^{\circ} \mathrm{C}-z$ min, where $x, y$ and $z$ stand for the rGO content in PVA, the reduction temperature and time respectively. By the way, in section 3.3, the total time means the whole reduction period for preparing the sample which includes the heating time and reduction time.

Furthermore, the effect of the networks formed by SrGO or LrGO sheets on the electrical conductivity is studied by swelling treatment. The method is immersing the nanocomposites films in reverse osmosis water (with the resistance of $18 \mathrm{M} \Omega$ ) for $15 \mathrm{~h}$ at room temperature, and then they are freezedried. It can be expected the network would be destroyed due to water swelling, and it causes the change of the electrical conductivity of the nanocomposites. So the above-mentioned effect may be revealed by the change of the electrical conductivity.

\subsection{Characterization}

The FEI Inspect F scanning electron microscopy (Inspect F, FEI Company, USA) with the an acceleration voltage of $5 \mathrm{kV}$ was used to measure the area of LGO and SGO sheets which were spincoated onto freshly cleaved ITO substrates, about 150 pieces of LGO or SGO sheets were measured. The resistance of the films was measured with Keithley 6487 electrometer (Keithley Instruments, Inc, Ohio, USA). Two-point method was used. Silver paint was applied to both ends of the sample to ensure good contact. As a result, contact resistance is negligible comparing to sample resistance. The bulk conductivity was calculated by Equation (1):

$\sigma=\frac{L}{R \cdot S}$

where $L, R$ and $S$ are the length, resistance and cross sectional area of the specimen, respectively. In addition, the electrical conductivity of nanocomposites was measured along the films. Previous researches indicated that the GO sheets tend to align parallel with the film surface [15], indicating that the charges are prone to transport along the films surface instead in perpendicular direction. So the electrical conductivity along the films surface is expected to be higher than that in perpendicular direction.

TGA (Q500, TA Instruments, USA) was performed under $\mathrm{N}_{2}$ from the room temperature to $260^{\circ} \mathrm{C}$ at a rate of $10^{\circ} \mathrm{C} / \mathrm{min}$. 


\section{Results and discussion}

\subsection{The decision of the reduction temperature}

TGA and DSC are used to characterize the thermal reduction processing of GO sheets (Figure 1). TGA result shows that LGO and SGO sheets exhibit a

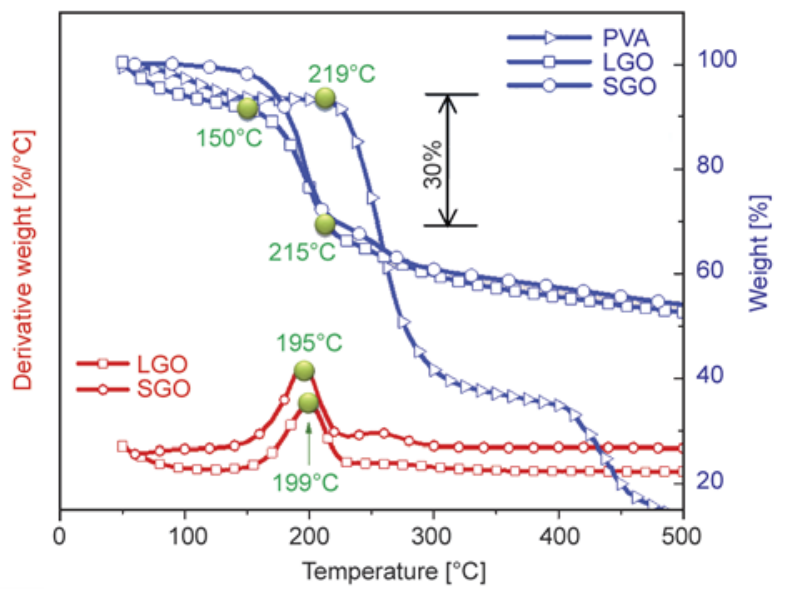

Figure 1. The TGA plots of PVA, LGO and SGO

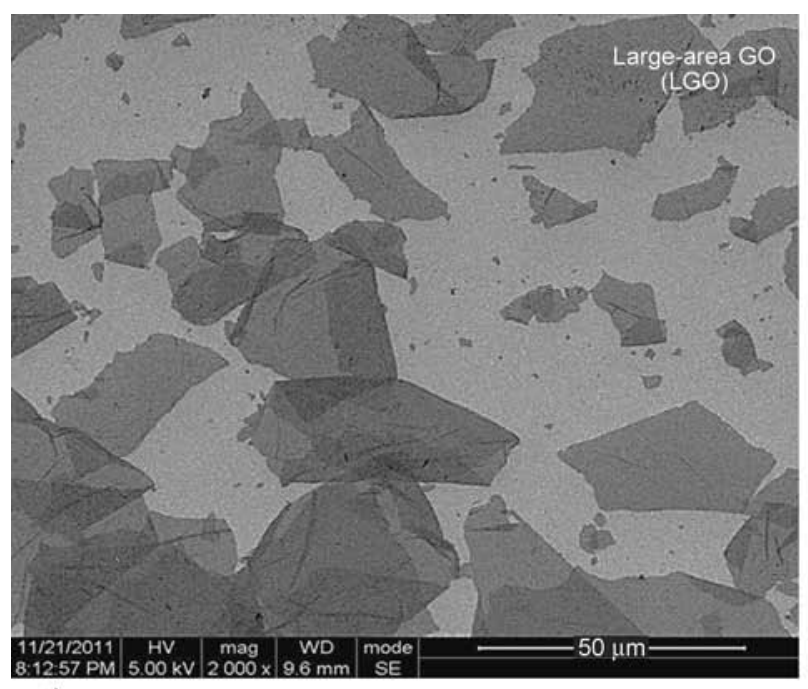

a)

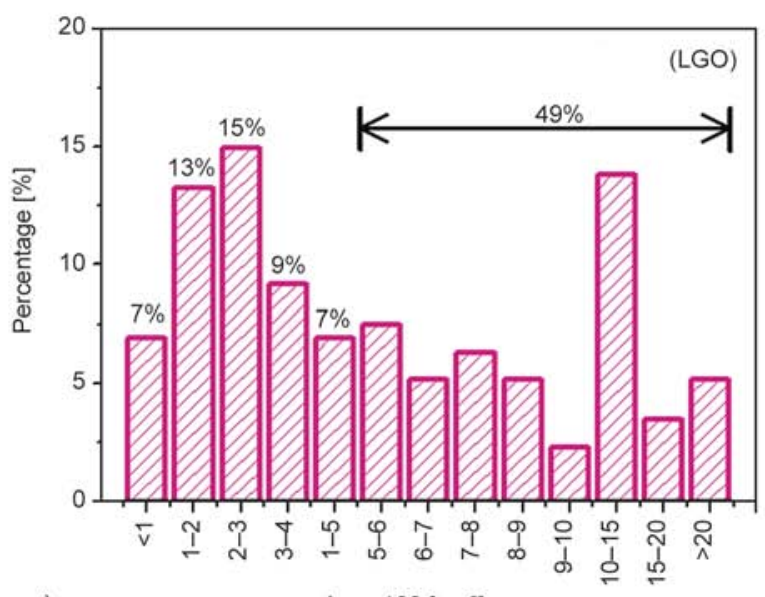

dramatic mass loss when reduction temperature reaches $215^{\circ} \mathrm{C}$. This indicates that the GO sheets lose some oxygen functional groups in the temperature range of $150 \sim 215^{\circ} \mathrm{C}$, and become the rGO sheets. The derivative weight curves of LGO and SGO sheets reveal that the strong reduction reaction occurs at the temperature of 195 and $199^{\circ} \mathrm{C}$. Due to the onset decomposition temperature of PVA matrix is $219^{\circ} \mathrm{C}$, the reduction temperature for reducing the PVA/GO nanocomposites are set as 150, 160, 170, 175,180 and $200^{\circ} \mathrm{C}$.

\subsection{The Properties of GO}

Figures $2 \mathrm{a}$ and $2 \mathrm{~b}$ show the typical SEM pictures of the LGO and SGO sheets. Based on the measurements of 150 pieces of LGO and SGO sheets, the respective area distribution is shown in Figures $2 \mathrm{c}$ and $2 \mathrm{~d}$. It can be clearly seen that about $49 \%$ of LGO sheets are larger than $500 \mu^{2}$, and $28 \%$ of LGO

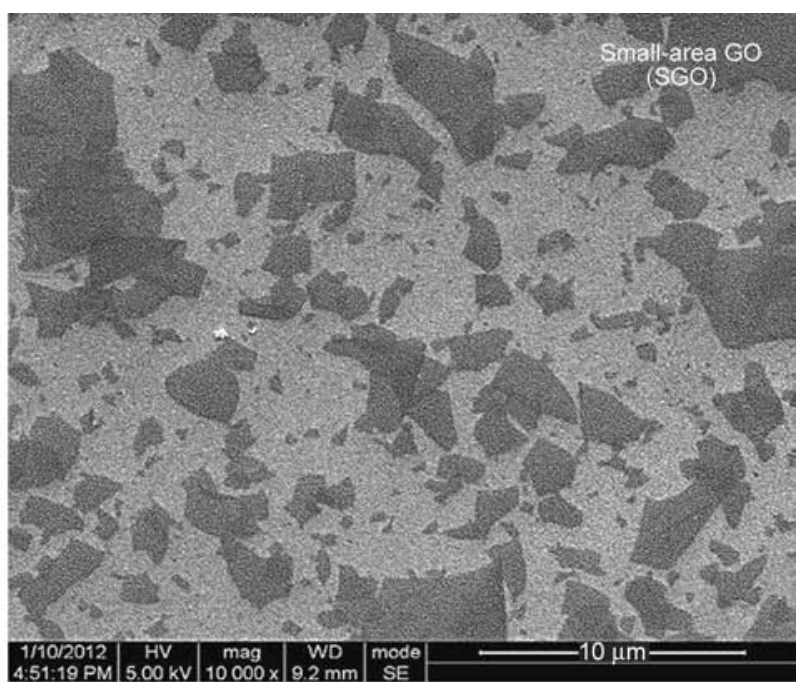

b)

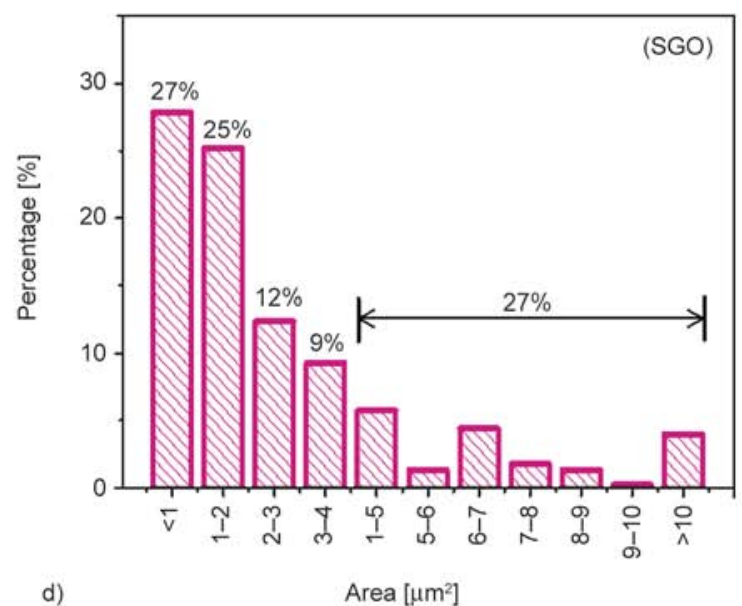

Figure 2. The SEM pictures of LGO (a) sheets and SGO sheets (b), which is used to count the area of LGO sheets (c) and SGO sheets (d) 
sheets has an area of ca.100 200 $\mu \mathrm{m}^{2}$. Due to the existence of weak $\mathrm{C}-\mathrm{O}-\mathrm{C}$ and stretched $\mathrm{C}-\mathrm{C}$ bonds on the surface of LGO sheets, the LGO sheets are broken into small ones after ultrasonic treatment, result $\sim 73 \%$ of SGO sheets with an area less than $5 \mu \mathrm{m}^{2}$ (Figures $2 \mathrm{~b}$ and $2 \mathrm{~d}$ ). After reduction, the resistance of $\mathrm{LrGO}$ film is $1.55 \mathrm{~K} \Omega /$ square which is lower than that of $\mathrm{SrGO}(8.97 \mathrm{~K} \Omega /$ square $)$, demonstrating that the LrGO sheets have better electrical conductivity and lower resistance than $\mathrm{SrGO}$ sheets [9].

\subsection{The electrical conductivity of the nanocomposites}

In our work, the electrical conductivity of the PVA/ LrGO nanocomposites is already $2.5 \cdot 10^{-6} \mathrm{~S} / \mathrm{m}$ at the LrGO content of $0.3 \mathrm{wt} \%$, which is higher than that of PVA/SrGO at the same filler content. Furthermore, the electrical conductivity of PVA/LrGO nanocomposites increases to $2.09 \cdot 10^{-4} \mathrm{~S} / \mathrm{m}$ with the addition of $1 \mathrm{wt} \% \mathrm{LrGO}$ (Figure $3 \mathrm{a}$ ). It indicates that the LrGO sheets could more efficiently improve the electrical conductivity of nanocomposites than SrGO sheets. The power law (see Equation (2)) is used to analyze electrical conductivity in order to reveal that the LrGO could decrease the percolation threshold of nanocomposites [18]:

$\sigma \propto \sigma_{0}\left(\varphi-\varphi_{\mathrm{c}}\right)^{v}$

where $\sigma_{0}$ is the bulk electrical conductivity of the fillers, $\varphi$ is the filler volume fraction, and $v$ is the universal critical exponent which depends on the dimensionality of the conductive network. The percolation threshold $\left(\varphi_{\mathrm{c}}\right)$ is the critical content above which a continuous connected network is formed for the transport of electrons throughout the matrix. Hence, it can be calculated that the $\varphi_{\mathrm{c}}$ value is 0.198 and $0.27 \mathrm{wt} \%$ for the sample of PVA $/ \mathrm{LrGO}-150^{\circ} \mathrm{C}$ $15 \mathrm{~min}$ and $\mathrm{PVA} / \mathrm{SrGO}-150^{\circ} \mathrm{C}-15$ min respectively (Figures $3 \mathrm{~b}$ and $3 \mathrm{c}$ ). Comparing with the percolation threshold reported in others' works (between $0.5 \sim 1 \mathrm{wt} \%$ ) $[19,20]$, the percolation threshold of $\mathrm{PVA} / \mathrm{LrGO}$ nanocomposites is only $0.198 \mathrm{wt} \%$. Besides that, the electrical conductivity of PVA/ LrGO-0.3 wt $\%-150^{\circ} \mathrm{C}-15 \mathrm{~min}$ is $2.5 \cdot 10^{-6} \mathrm{~S} / \mathrm{m}$, better than that of $\mathrm{PVA} / \mathrm{rGO}$ nanocomposites reported in refs. [19] and [20], in which the electrical conductivity of nanocomposites was below $10^{-12} \mathrm{~S} / \mathrm{m}$ at the $0.3 \mathrm{wt} \% \mathrm{rGO}$ contents. In summary, the LrGO sheets not only decrease the percolation threshold,
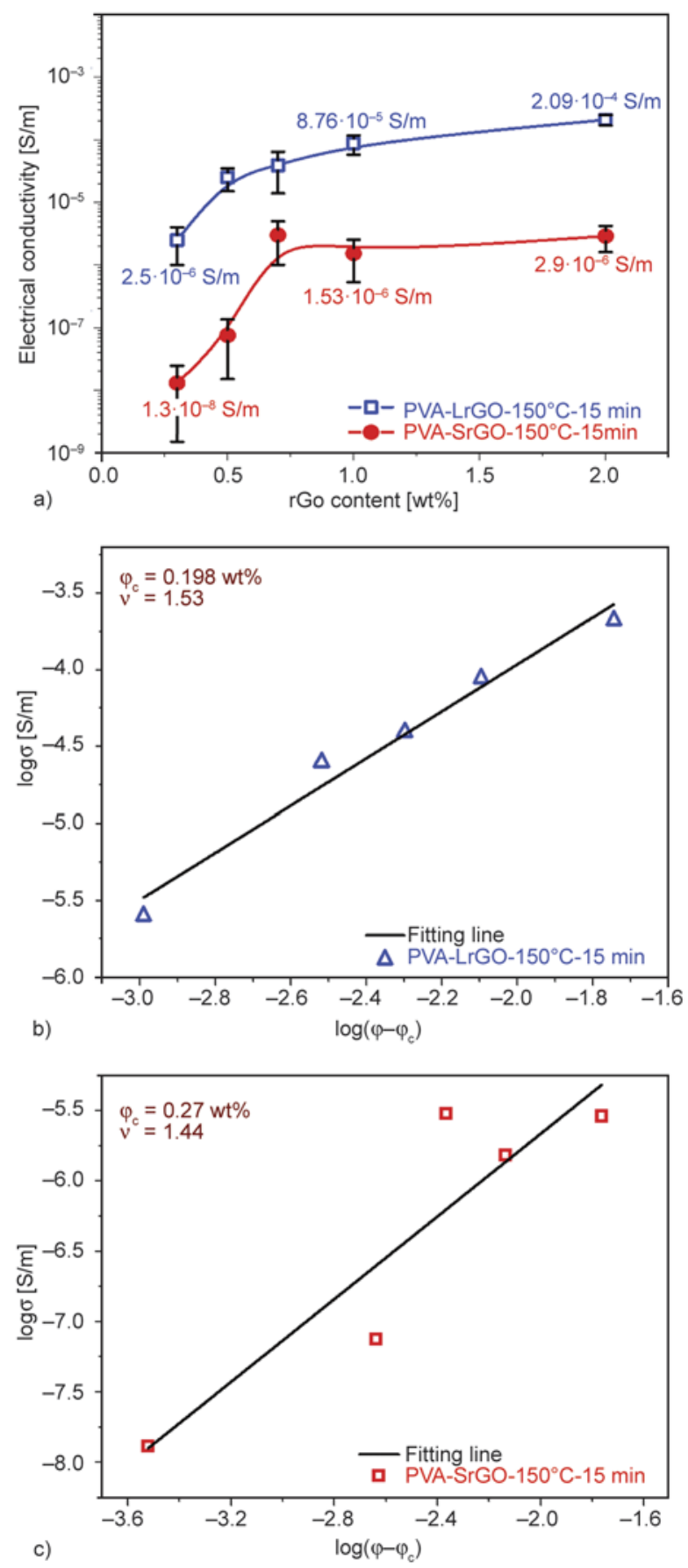

Figure 3. a) The electrical conductivity of PVA-LrGO$150^{\circ} \mathrm{C}-15 \mathrm{~min}$ and PVA-SrGO- $150^{\circ} \mathrm{C}-15 \mathrm{~min}$ as a function of the rGO content. b) and c) are the double-logarithmic plot of electrical conductivity versus $\left(\varphi-\varphi_{\mathrm{c}}\right)$ for the samples of PVA-LrGO$150^{\circ} \mathrm{C}-15 \mathrm{~min}$ and PVA-SrGO- $150^{\circ} \mathrm{C}-15 \mathrm{~min}$

but also could improve the electrical conductivity of nanocomposites especially at a low content of LrGO.

Moreover, the high reduction temperature could make the nanocomposites films having high electrical conductivity. The electrical conductivity of PVA/ LrGO nanocomposites increases more remarkably 
than those of PVA/SrGO nanocomposites. As shown in Figure $4 \mathrm{a}$, the electrical conductivity of PVA/ LrGO greatly increased from $9.8 \cdot 10^{-8}$ to $6.3 \cdot 10^{-3} \mathrm{~S} / \mathrm{m}$ with increasing the reduction temperature. The electrical conductivity increased to the highest value at the reduction temperature of $200^{\circ} \mathrm{C}$, indicating that the critical temperature for the thermal reduction in PVA/GO system is $200^{\circ} \mathrm{C}$. According to results of derivative weight, it can be known that the critical temperature is the reduction temperature of GO. So when the thermal reduction temperature exceeds the critical temperature, the electrical conductivity of nanocomposites can be improved remarkably, otherwise, it should be not.

The Figures $4 \mathrm{~b}$ and $4 \mathrm{c}$ show the change of electrical conductivity as increasing the reduction time at low temperature and high temperature respectively. Under the same reduction time, the electrical conductivity of the sample reduced at high reduction temperature is always higher than that reduced at low reduction temperature (Figures $4 \mathrm{a}$ and $4 \mathrm{c}$ ), indicating the high temperature is more efficient for reducing the GO sheets than low temperature. The long reduction time also could improve the electrical conductivity of nanocomposites (Figures $4 \mathrm{~b}$ and $4 \mathrm{c}$ ). For example (Table 1), the final electrical conductivities of the PVA/LrGO-0.7 wt $\%-200^{\circ} \mathrm{C}-0$ min and PVA/LrGO$0.7 \mathrm{wt} \%-150^{\circ} \mathrm{C}-30 \mathrm{~min}$ are much closer; but the total time (including the heating time and reduction time) of former is $17 \mathrm{~min}$ shorter than that of latter (42 $\mathrm{min}$ ). Therefore from the perspective of reduction efficiency, the high reduction temperature is a more efficient way to improve electrical conductivity of nanocomposites in comparison with extending the reduction time.

Table 2 is listed for comparing the final electrical conductivity of nanocomposites prepared by our method with that reported by others, in which the rGO sheets were re-dispersed by surfactant agents. The results show that the electrical conductivity reported by us is $6.3 \cdot 10^{-3} \mathrm{~S} / \mathrm{m}$ higher than others' at the rGO content of $0.3 \sim 0.7 \mathrm{wt} \%[19,20]$, the electrical conductivity of them did not being mentioned or is about $10^{-5} \mathrm{~S} / \mathrm{cm}$ at $1 \mathrm{wt} \% \mathrm{rGO}$ content $[14,20]$. Due to that the surfactant agents hinder the overlap between rGO sheets [21], increase the contact resistance. So the thermal reduction could get rid of using surfactant and maintain the low contact resistance between the interlayer of rGO sheets. Besides that, this method only takes $15 \sim 30$ min which is shorter
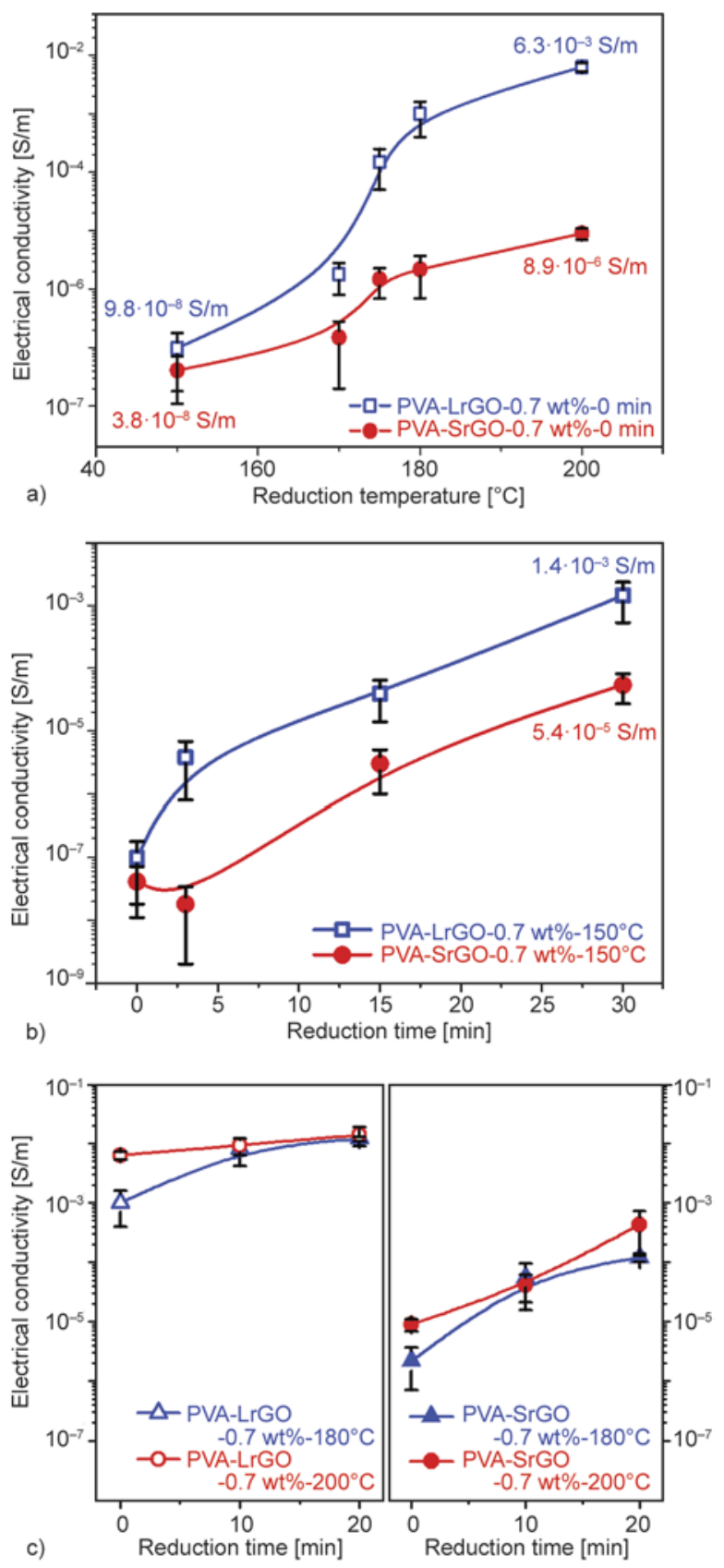

Figure 4. a) The electrical conductivity of PVA-LrGO$0.7 \mathrm{wt} \%-0 \mathrm{~min}$ and PVA-SrGO-0.7 $\mathrm{wt} \%-0 \mathrm{~min}$ as a function of the reduction temperature, $b$ ) the electrical conductivity of PVA-LrGO-0.7 wt $\%$ $150^{\circ} \mathrm{C}$ and PVA-SrGO- 0.7 wt. $\%-150^{\circ} \mathrm{C}$ as a function of the reduction time, and c) the electrical conductivity of PVA-LrGO-0.7 wt $\%-180^{\circ} \mathrm{C}$, PVALrGO-0.7 wt $\%-200^{\circ} \mathrm{C}$, PVA-SrGO- $0.7 \mathrm{wt} \%-180^{\circ} \mathrm{C}$ and PVA-SrGO- $0.7 \mathrm{wt} \%-200^{\circ} \mathrm{C}$ as a function of the reduction time

than present chemical reduction process (takes $24 \mathrm{~h}$ $[15,20])$. In summary, the thermal reduction is an efficient way to prepare the nanocomposites with low percolation threshold and high electrical conductivity, and also is suitable for preparing the ther- 
Table 1.The electrical conductivity of PVA/rGO nanocomposites and the total time for preparing them

\begin{tabular}{|l|c|c|c|c|}
\hline \multicolumn{1}{|c|}{ Sample } & $\begin{array}{c}\text { Electrical conductivity } \\
{[\mathbf{S} / \mathbf{m}]}\end{array}$ & $\begin{array}{c}\text { Heating time } \\
{[\mathbf{m i n}]}\end{array}$ & $\begin{array}{c}\text { Reduction time } \\
{[\mathbf{m i n}]}\end{array}$ & $\begin{array}{c}\text { Total time } \\
{[\mathbf{m i n}]}\end{array}$ \\
\hline PVA/LrGO-0.7 $\mathrm{wt} \%-200^{\circ} \mathrm{C}-0 \mathrm{~min}$ & $6.3 \cdot 10^{-3}$ & 17 & 0 & 17 \\
\hline PVA/LrGO-0.7 $\mathrm{wt} \%-200^{\circ} \mathrm{C}-10 \mathrm{~min}$ & $9.2 \cdot 10^{-3}$ & 17 & 10 & 27 \\
\hline PVA/LrGO-0.7 $\mathrm{wt} \%-150^{\circ} \mathrm{C}-0 \mathrm{~min}$ & $9.8 \cdot 10^{-8}$ & 12 & 0 & 12 \\
\hline PVA/LrGO-0.7 $\mathrm{wt} \%-150^{\circ} \mathrm{C}-30 \mathrm{~min}$ & $1.4 \cdot 10^{-3}$ & 12 & 30 & 42 \\
\hline PVA/SrGO-0.7 $\mathrm{wt} \%-200^{\circ} \mathrm{C}-0 \mathrm{~min}$ & $8.9 \cdot 10^{-6}$ & 17 & 0 & 17 \\
\hline PVA/SrGO-0.7 $\mathrm{wt} \%-200^{\circ} \mathrm{C}-10 \mathrm{~min}$ & $1.4 \cdot 10^{-5}$ & 17 & 10 & 27 \\
\hline PVA/SrGO-0.7 $\mathrm{wt} \%-150^{\circ} \mathrm{C}-0 \mathrm{~min}$ & $3.8 \cdot 10^{-8}$ & 12 & 0 & 12 \\
\hline PVA/SrGO-0.7 $\mathrm{wt} \%-150^{\circ} \mathrm{C}-30 \mathrm{~min}$ & $5.4 \cdot 10^{-5}$ & 12 & 30 & 42 \\
\hline
\end{tabular}

Table 2. The electrical conductivity of PVA/rGO nanocomposites comparing between our results and literature $[14,15,19,20]$

\begin{tabular}{|c|c|c|c|c|c|}
\hline rGO content & Surfactant & $\begin{array}{c}\text { Electrical } \\
\text { conductivity }\end{array}$ & $\begin{array}{c}\text { Reduced agents or } \\
\text { method }\end{array}$ & Reduction Time & Reference \\
\hline $3 \mathrm{vol} \%$ & SDBS & - & Hydrazine & $30 \mathrm{~min}$ & 14 \\
\hline $1.6 \mathrm{wt} \%$ & - & $10^{-9} \mathrm{~S} / \mathrm{cm}$ & Hydrazine & 24 hour & 20 \\
\hline $3.5 \mathrm{wt} \%$ & - & - & Hydrazine & 24 hour & 15 \\
\hline $1 \mathrm{wt} \%$ & $2-$ propanol & $10^{-5} \mathrm{~S} / \mathrm{cm}$ & Hydrazine & Overnight & 19 \\
\hline $0.7 \mathrm{wt} \%$ & - & $6.3 \cdot 10^{-3} \mathrm{~S} / \mathrm{m}$ & Thermal reduction at $200^{\circ} \mathrm{C}$ & $17 \mathrm{~min}$ & Our work \\
\hline
\end{tabular}

moset or thermoplastic polymer/rGO nanocomposites through reducing the GO sheets at appropriate temperature.

\subsection{The structure of the electrical networks}

The mechanism of electrical conductivity of the nanocomposites is based on two factors. The first one is the intrinsic electrical conductivity of nanofiller. In this system, the electrical conductivity of LrGO sheets is higher than that of SrGO sheets, it could affect the final electrical conductivity of nanocomposites, but the interesting thing is that the resistance difference between $\mathrm{LrGO}$ sheets $(1.55 \mathrm{~K} \Omega$ /square) and $\mathrm{SrGO}(8.97 \mathrm{~K} \Omega$ /square) sheets is only one order of magnitude while the electrical conductivity dif- ference between the LrGO/PVA and SrGO/PVA nanocomposites is two or three orders of magnitude under the same reduced condition. This question would be answered through analyzing the structure of electrical conductive network formed by LrGO or SrGO sheets in PVA matrix.

The swelling treatment is used to study the effect of the SrGO and LrGO sheets on the electrical conductive network morphology in nanocomposites. Figure 5 shows that the electrical conductivity of the nanocomposites decreased after swelling. The electrical conductivity of $\mathrm{PVA} / \mathrm{LrGO}-1 \mathrm{wt} \%-180^{\circ} \mathrm{C}$ $10 \mathrm{~min}$ is decreased from $3.4 \cdot 10^{-2}$ to $3.6 \cdot 10^{-4} \mathrm{~S} / \mathrm{m}$ (Figure 5a); and that of PVA/SrGO- $1 \mathrm{wt} \%-180^{\circ} \mathrm{C}$ $10 \mathrm{~min}$ also decrease from $2.1 \cdot 10^{-4}$ to $1.77 \cdot 10^{-7} \mathrm{~S} / \mathrm{m}$
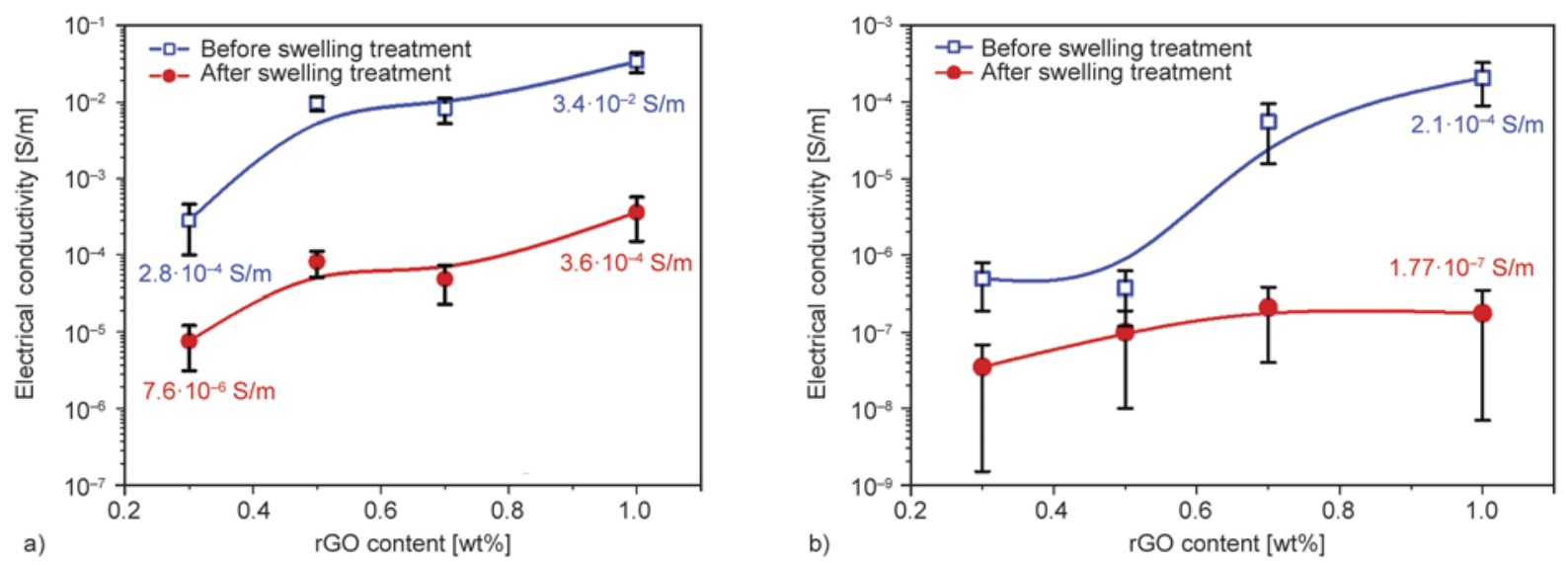

Figure 5. The change of the electrical conductivity of PVA-LrGO- $180^{\circ} \mathrm{C}-10 \mathrm{~min}$ (a) and PVA-SrGO- $180^{\circ} \mathrm{C}-10 \mathrm{~min}$ (b) before (blue curve) and after (red curve) swelling treatment 
(Figure 5b). Because the electrical conductivity of LrGO or SrGO sheets in nanocomposites doesn't change during the swelling treatment, so the decreased electrical conductivity of nanocomposites may be due to the destroyed conductive networks. During the swelling treatment, water molecules could intercalate between the rGO sheets, open the contact points of rGO sheets, and then destroy the electrically conductive network, causing the decrease of the electrical conductivity of the nanocomposites. Figure 5 shows that the decrease of electrical conductivity of PVA/LrGO nanocomposites is less than that of PVA/SrGO nanocomposites after the swelling treatment, indicating that the network formed by LrGO sheets is firmer than that formed by $\mathrm{SrGO}$ sheets, and could not be destroyed easily by swelling treatment. Besides that, the LrGO sheets are prone to overlap with each other due to their large aspect ratio, and form a continuous conductive network at low filler content, causing the $\mathrm{PVA} / \mathrm{LrGO}$ nanocomposites having low percolation threshold.

\section{Conclusions}

This is an efficient method to preparing the low percolation threshold and high electrical conductivity PVA/rGO nanocomposites by using large area GO sheets. The LGO sheets would recover their electrical conductivity efficiently after being reduced due to the low degree of oxidation. The LrGO sheets could construct a continuous and firm network in PVA matrix due to their high aspect ratio. The formed continuous electrically conductive network could remarkably improve the electrical conductivity of PVA/LrGO nanocomposites. Besides, the thermal reduction process is a fast method for preparing electrical conductive polymer/LrGO nanocomposites which only takes 15 30 min. This method solves four problems existing in the process of preparing nanocomposites, which are 1) avoiding using the surfactant to redisperse the rGO sheets, 2) shortening the preparation period, 3) simplifying the posttreatment, 4) as well as increasing the electrical conductivity of nanocomposites at low rGO contents.

\section{References}

[1] Li N., Cheng W., Ren K., Luo F., Wang K., Fu Q.: Oscillatory shear-accelerated exfoliation of graphite in polypropylene melt during injection molding. Chinese Journal of Polymer Science, 31, 98-109 (2013). DOI: $10.1007 / \mathrm{s} 10118-013-1204-0$

[2] Lin L., Deng H., Gao X., Zhang S., Bilotti E., Peijs T., Fu Q.: Modified resistivity-strain behavior through the incorporation of metallic particles in conductive polymer composite fibers containing carbon nanotubes. Polymer International, 62, 134-140 (2013).

DOI: $10.1002 /$ pi.4291

[3] Gao X., Zhang S., Mai F., Lin L., Deng Y., Deng H., $\mathrm{Fu}$ Q.: Preparation of high performance conductive polymer fibres from double percolated structure. Journal of Materials Chemistry, 21, 6401-6408 (2011). DOI: 10.1039/C0JM04543H

[4] Long G., Tang C., Wong K-W., Man C., Fan M., Lau W-M., Xu T., Wang B.: Resolving the dilemma of gaining conductivity but losing environmental friendliness in producing polystyrene/graphene composites via optimizing the matrix-filler structure. Green Chemistry, 15, 821-828 (2013).

DOI: $10.1039 / \mathrm{c} 3 \mathrm{gc} 37042 \mathrm{a}$

[5] Kovacs J. Z., Velagala B. V., Schulte K., Bauhofer W.: Two percolation thresholds in carbon nanotube epoxy composites. Composites Science and Technology, 67, 922-928 (2007).

DOI: 10.1016/j.compscitech.2006.02.037

[6] Zhang S. M., Lin L., Deng H., Gao X., Bilotti E., Peijs T., Zhang Q., Fu Q.: Synergistic effect in conductive networks constructed with carbon nanofillers in different dimensions. Express Polymer Letters, 6, 159-168 (2012).

DOI: 10.3144/expresspolymlett.2012.17

[7] Novoselov K. S., Geim A. K., Morozov S. V., Jiang D., Zhang Y., Dubonos S. V., Grigorieva I. V., Firsov A. A.: Electric field effect in atomically thin carbon films. Science, 306, 666-669 (2004). DOI: $10.1126 /$ science. 1102896

[8] Grossiord N., Loos J., Regev O., Koning C. E.: Toolbox for dispersing carbon nanotubes into polymers to get conductive nanocomposites. Chemistry of Materials, 18, 1089-1099 (2006). DOI: $10.1021 / \mathrm{cm} 051881 \mathrm{~h}$

[9] Zhao J., Pei S., Ren W., Gao L., Cheng H-M.: Efficient preparation of large-area graphene oxide sheets for transparent conductive films. ACS Nano, 4, 52455252 (2010). DOI: $10.1021 / \mathrm{nn} 1015506$

[10] Liao K-H., Mittal A., Bose S., Leighton C., Mkhoyan K. A., Macosko C. W.: Aqueous only route toward graphene from graphite oxide. ACS Nano, 5, 12531258 (2011). DOI: $\underline{10.1021 / \mathrm{nn} 1028967}$ 
[11] Nethravathi C., Rajamathi M.: Chemically modified graphene sheets produced by the solvothermal reduction of colloidal dispersions of graphite oxide. Carbon, 46, 1994-1998 (2008).

DOI: $10.1016 /$ j.carbon.2008.08.013

[12] Glover A. J., Cai M., Overdeep K. R., Kranbuehl D. E., Schniepp H. C.: In situ reduction of graphene oxide in polymers. Macromolecules, 44, 9821-9829 (2011). DOI: $10.1021 / \mathrm{ma} 2008783$

[13] Tang H., Ehlert G. J., Lin Y., Sodano H. A.: Highly efficient synthesis of graphene nanocomposites. Nano Letters, 12, 84-90 (2011).

DOI: $10.1021 / \mathrm{nl} 203023 \mathrm{k}$

[14] Zhao X., Zhang Q., Chen D., Lu P.: Enhanced mechanical properties of graphene-based poly(vinyl alcohol) composites. Macromolecules, 43, 2357-2363 (2010). DOI: 10.1021/ma902862u

[15] Yang X., Li L., Shang S., Tao X-M.: Synthesis and characterization of layer-aligned poly(vinyl alcohol)/ graphene nanocomposites. Polymer, 51, 3431-3435 (2010). DOI: 10.1016/j.polymer.2010.05.034

[16] Zheng D., Tang G., Zhang H-B., Yu Z-Z., Yavari F., Koratkar N., Lim S-H., Lee M-W.: In situ thermal reduction of graphene oxide for high electrical conductivity and low percolation threshold in polyamide 6 nanocomposites. Composites Science and Technology, 72, 284-289 (2012).

DOI: $10.1016 /$ j.compscitech.2011.11.014
[17] Hummers W. S., Offeman R. E.: Preparation of graphitic oxide. Journal of the American Chemical Society, 80, 1339 (1958).

DOI: $10.1021 / \mathrm{ja} 01539 \mathrm{a} 017$

[18] Kovacs J. Z., Velagala B. S., Schulte K., Bauhofer W.: Two percolation thresholds in carbon nanotube epoxy composites. Composites Science and Technology, 67, 922-928 (2007).

DOI: 10.1016/j.compscitech.2006.02.037

[19] Salavagione H. J., Martínez G., Gómez M. A.: Synthesis of poly(vinyl alcohol)/reduced graphite oxide nanocomposites with improved thermal and electrical properties. Journal of Materials Chemistry, 19, 5027-5032 (2009).

DOI: $10.1039 / \mathrm{B} 904232 \mathrm{~F}$

[20] Bao C., Guo Y., Song L., Hu Y.: Poly(vinyl alcohol) nanocomposites based on graphene and graphite oxide: A comparative investigation of property and mechanism. Journal of Materials Chemistry, 21, 1394213950 (2011).

DOI: 10.1039/C1JM11662B

[21] Wang J., Zhou T., Deng H., Chen F., Wang K., Zhang Q., Fu Q.: An environmentally friendly and fast approach to prepare reduced graphite oxide with water and organic solvents solubility. Colloids and Surfaces B: Biointerfaces, 101, 171-176 (2013). DOI: $10.1016 /$ j.colsurfb.2012.06.008 